Kompass

Autoimmun

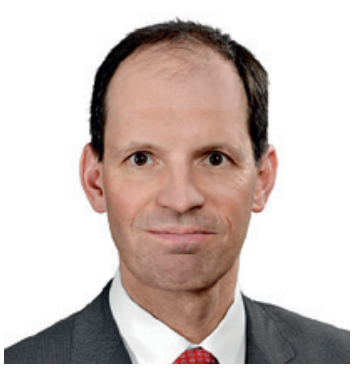

\title{
Erste EULAR-Empfehlungen (Points to Consider) für rheumatische Nebenwirkungen von Checkpoint-Inhibitoren
}

Martin Aringer

Universitätsklinikum Carl Gustav Carus der Technischen Universität Dresden, Medizinische Klinik und Poliklinik III, Dresden, Deutschland

Abstract aus Kostine M, Finckh A, Bingham CO, et al.: EULAR points to consider for the diagnosis and management of rheumatic immune-related adverse events due to cancer immunotherapy with checkpoint inhibitors. Ann Rheum Dis 2021;80:36-48.

\section{Keywords}

Arthritis · Autoimmunity · Inflammation · Multidisciplinary team care Treatment

\begin{abstract}
Background: Rheumatic and musculoskeletal immune-related adverse events (irAEs) are observed in about 10\% of patients with cancer receiving checkpoint inhibitors (CPIs). Given the recent emergence of these events and the lack of guidance for rheumatologists addressing them, a European League Against Rheumatism task force was convened to harmonise expert opinion regarding their identification and management.
\end{abstract}

Methods: First, the group formulated research questions for a systematic literature review. Then, based on literature and using a consensus procedure, 4 overarching principles and 10 points to consider were developed.

Results: The overarching principles defined the role of rheumatologists in the management of irAEs, highlighting the shared decision-making process between patients, oncologists and rheumatologists. The points to consider inform rheumatologists on the wide spectrum of musculoskeletal irAEs, not fulfilling usual classification criteria of rheumatic diseases, and their differential diagnoses. Early referral and facilitated access to rheumatologist are recommended, to document the target organ inflammation. Regarding therapeutic, three treatment escalations were defined: (1) local/systemic glucocorticoids if symptoms are not controlled by symptomatic treatment, then tapered to the lowest efficient dose, (2) conventional synthetic disease-modifying antirheumatic drugs, in case of inadequate response to glucocorticoids or for steroid sparing and (3) biological disease-modifying antirheumatic drugs, for severe or refractory irAEs. A warning has been made on severe myositis, a life-threatening situation, requiring high dose of glucocorticoids and close monitoring. For patients with pre-existing rheumatic disease, baseline immunosuppressive regimen should be kept at the lowest efficient dose before starting immunotherapies.

Conclusion: These statements provide guidance on diagnosis and management of rheumatic irAEs and aim to support future international collaborations.

(c) 2021 The authors. Published by BMJ. 


\section{Transfer in die Praxis}

\section{Hintergrund}

Die Gruppe der Checkpoint-Inhibitoren, PD-1-Hemmer (Nivolumab, Pembrolizumab, Cemiplimab), PD-L1-Hemmer (Atezolizumab, Avelumab, Durvalumab) und der CTLA-4-Blocker Ipilimumab, kann bei Melanomen und anderen malignen Tumoren durch T-Zell-Aktivierung vorher nicht denkbare Therapieerfolge erzielen. Je mehr sie daher eingesetzt werden, desto häufiger werden auch unerwünschte immunologische Wirkungen (immune-related adverse events, irAEs). Diese irAEs können alle Organe betreffen, wie bei der Graft-versus-Host Disease (GvHD) nach allogener Stammzelltransplantation besonders häufig Haut, Gastrointestinaltrakt und Lunge, aber auch endokrine Drüsen. Etwa 10\% dieser Patienten dürften aber (atypische) rheumatische Manifestationen entwickeln, die von Arthralgien und Myalgien über Arthritiden und Polymyalgia rheumatica-ähnliche Symptomatik bis zu lebensbedrohlichen Myositiden gehen. Die European League Against Rheumatism (EULAR) hat jetzt erstmals auf Basis der begrenzten Literatur und eines Expertenkonsens Points to Consider (in etwa: «Relevante Aspekte») als niedrigste Empfehlungsstufe herausgegeben. Wie bei den meisten EULAR-Dokumenten zahlt es sich aus, sie im Detail zu lesen.

\section{Wesentliche Inhalte der Points to Consider}

Ganz wichtig ist bei dieser Fragestellung die Grundkonzeption, die auch in den Overarching Principles ausgeführt wird: Der Kampf gegen den Krebs hat in aller Regel weiter Vorrang, daher muss die Abstimmung nicht nur (wie immer in der Rheumatologie) mit den Patient*innen, sondern auch mit den Onkolog*innen sehr eng sein. Das Ziel ist daher auch nicht die komplette Remission der Symptome, sondern eine für die individuellen Patient*innen (gut) erträgliche Situation. In die gleiche Richtung geht die Empfehlung, Patient*innen mit entzündlich-rheumatischen Erkrankungen nicht von Therapien mit Checkpoint-Inhibitoren auszuschließen. Schübe sind nicht die Regel und meist gut kontrollierbar.

Auch wenn die Datenlage insgesamt begrenzt ist, scheinen Glukokortikoide bis $10 \mathrm{mg}$ Prednisolon täglich (und intraartikuläre Glukokortikoide) den Antitumor-Effekt nicht wesentlich zu hemmen, während eine Glukokortikoid-Bolustherapie tumorimmunologisch kritisch ist. Sind die Glukokortikoide nicht in diesen Bereich zu reduzieren, kommen immunmodulierende Basistherapeutika zum Einsatz. Hydroxychloroquin und Methotrexat gelten dabei als relativ unproblematisch, während es unter Sulfasalazin vermehrt allergische Reaktionen gibt. Auf der Ebene der Biologika kommen TNFBlocker und Interleukin-6-Rezeptor-Blocker infrage.
Die EULAR befürwortet, Rheumatolog*innen rasch hinzuzuziehen. Die Points to Consider weisen darauf hin, dass neben den irAEs auch Metastasen, paraneoplastische Erkrankungen wie die hypertrophe Osteoarthropathie (Pierre-Marie-Bamberger) und völlig unabhängige Probleme infrage kommen. Unter Nivolumab sind zum Beispiel rezidivierende Episoden einer Pseudogicht (Kalziumpyrophosphat) aufgetreten. Gerade bei Monarthritiden ist die Punktion daher auch diagnostisch sinnvoll.

Polymyalgie-artige Krankheitsbilder treten häufig relativ früh (60 Tage) unter Checkpoint-Inhibitor-Therapie auf, verschiedene Formen einer eindeutig nachweisbaren Arthritis im Mittel nach doppelt so langer Therapie. Seltener sind Vaskulitiden und Kollagenose-artige Symptome, insbesondere auch der Haut - zum Beispiel Sklerodermie- oder auch SCLE-artig (SCLE = subakut kutaner Lupus erythematodes). Die typischen Autoantikörper sind meist, in etwa 4 von 5 Fällen, negativ - und höhertitrige antinukleäre Antikörper (ANA) sind ebenso ungewöhnlich. Es wird auch nicht empfohlen, sie im Vorfeld zu bestimmen.

Dass die ANA als Marker oft nicht helfen, gilt auch für die Sjögrenartige Sicca-Symptomatik, die auch gepaart mit neurologischen Symptomen auftreten kann. Dieses Krankheitsbild unterscheidet sich allerdings auch histologisch durch ausgeprägtere T-Zell-Infiltrate.

Als eigener Punkt wird auf schwer verlaufende proximale Myositiden mit entweder bulbärer Symptomatik (und Kombinationen mit einer Myasthenia gravis) oder mit Myokarditiden hingewiesen. Dann besteht Lebensgefahr und eine Unterbrechung der Checkpoint-Inhibitoren ist notwendig. Hier kommen auch höher dosierte Glukokortikoide, intravenöse Immunglobuline und/oder Plasmapherese und Mycophenolat zum Einsatz, und Abatacept und Alemtuzumab wurden in Einzelfällen versucht.

\section{Fazit für die Praxis}

Die irAEs bei Checkpoint-Inhibitoren werden in Anbetracht der Erfolge dieser Substanzgruppe weiter zunehmen. Auf einer noch relativ dünnen Evidenzbasis geben die EULAR Points to Consider jetzt erstmals gute, konkrete Ratschläge für eine individuell immer schwierige Situation.

\section{Disclosure Statement}

Advisory Boards und/oder Vorträge Astra Zeneca, BMS, MSD, Roche, Sanofi.

Korrespondenz an:

Prof. Dr. Martin Aringer, martin.aringer@uniklinikum-dresden.de 